\title{
Advocating for Diversity and Inclusion in Faculty Hiring
}

\section{Darryl Yong and Sumun Pendakur}

Note: The opinions expressed here are not necessarily those of Notices.

Responses on the Notices webpage are invited.

EDITOR'S NOTE: Yong and Pendakur have kindly provided these recommended best practices for diversity and inclusion in faculty hiring.

The mathematical sciences have a longstanding and persistent history of underrepresentation of women and certain ethnic groups, in particular, African American/Black, Hispanic/LatinX, Native American, Alaska

Native, and Pacific Islander individuals. ${ }^{1}$ One potentially fruitful way to broaden participation in the mathematical sciences is to adopt faculty hiring practices that promote diversity and inclusion, since personnel decisions are so important and long lasting. ${ }^{2}$

Many of us faculty are currently serving and will soon serve on hiring committees at our institutions. The goal of this article is to provide suggestions and resources on how to advocate for diversity and inclusion during the faculty-search process. In Sections 1 and 2, we provide some of these suggestions for

Darryl Yong is professor of mathematics at Harvey Mudd College and director of the Claremont Colleges Center for Teaching and Learning. His e-mail address is dyong@hmc.edu.

Sumun Pendakur EdD is associate dean for institutional diversity at Harvey Mudd College. Her e-mail address is spendakur@hmc . edu .

${ }^{1}$ Data can be found in various sources, such as the NSF, NCES, and AMS Annual Survey of the Mathematical Sciences www . ams . org/ profession/data/annual-survey/annual-survey.

${ }^{2}$ We seek to promote all forms of diversity, including gender, ethnicity, age, religion, and sexual orientation, but the aforementioned underrepresentation compels us to acknowledge the primacy of gender and ethnicity in this discussion. search committees and individuals serving on these committees. We end with some helpful resources in Section 3.

Though we have tried to give ideas that will apply to most people, we recognize that this article is a product of our experiences working in private institutions in California. Our hope is that committees and individuals will consider these ideas and adapt them to fit their own contexts. You should seek professional advice from human resource professionals or legal counsel if you have questions about what is permissible in your locality.

\section{Section 1. Best Practices for Search Committees}

Agree on departmental definitions and goals for diversity. Ultimately, success at increasing diversity depends on whether the people at the institution see diversity as important and connected to their institution's mission [5, 7]. There are many reasons why diversity is important to our institutions, to our profession, and to our nation. Ask yourself which of those reasons most strongly resonate with the mission statement of your institution. When we (individually and collectively) can clearly articulate reasons for promoting diversity, our efforts to promote diversity become more focused and effective. If those reasons also align with the missions of our institutions, then we can activate more colleagues to join in the effort.

Consequently, one of the most important steps that departments can take to support diversity in faculty hiring is to come to a shared understanding about what

For permission to reprint this article, please contact: reprint-permission@ams.org.

DOI: http://dx.doi.org/10.1090/noti1559 
is meant by words like "diversity" and "inclusion" and how those things relate to the mission of the department or institution. If your institution has a strong vision for diversity, that is a good place to start. Coming up with a departmental diversity vision or mission statement can be helpful, but written documents like these are helpful only insofar as they are supported and enacted by individuals in the department. The dialogue necessary to come to a shared understanding about diversity and inclusion is often more important than any document that is generated. This work might seem unrelated to faculty searches, but it isn't! Think of it as preparing a strong foundation for all of the work that your committee will do.

Seek administrative support. Diversity, inclusion, and equitable student outcomes are important concerns at many colleges and universities in the United States these days. Seek out administrators (department chairs, deans, chief diversity officers, human resource professionals, staff in your institution's multi/cross-cultural center) who will support your department in its commitment to diversity. Determine which hiring practices have been used by your institution and talk to other faculty members who have participated in successful hiring committees to determine if they encountered any institutional barriers and how they overcame them.

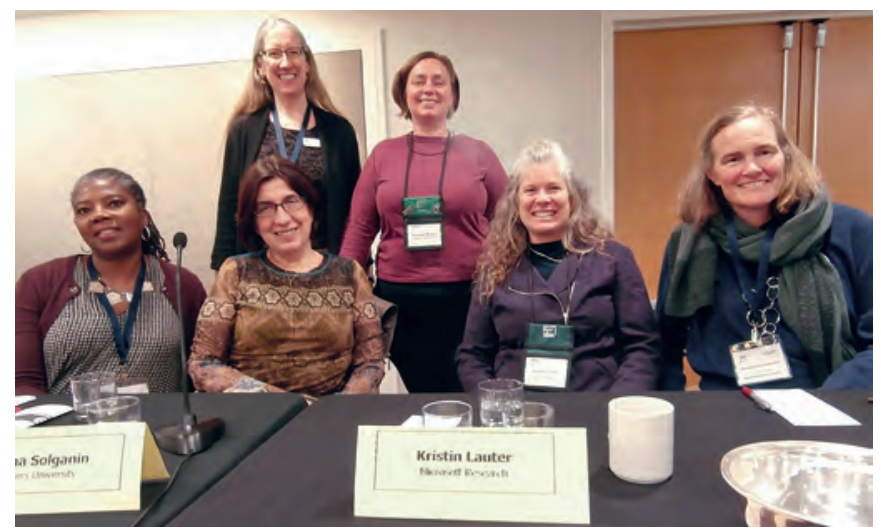

Association for Women in Mathematics (AWM) panel "Mentoring Women in Mathematics" at JMM 2017. Standing Helen Grundman, Michelle Manes; seated Suzanne Weekes, Emina Soljanin, Kristin Lauter, and Deanna Haunsperger.

Build your search committee thoughtfully, since its members will be doing most of the work in the hiring process. Each committee member has their own lens through which candidates are viewed. Therefore, to get the most comprehensive, holistic view of your candidates, it helps to have some diversity on the search committee itself. However, also be careful not to overtax faculty of color and women-research has shown that they tend to get asked to do a greater proportion of service responsibilities than others [3, 8]. If possible, choose the chair of this search committee carefully as that person will likely set the tone for the committee and model the behavior that others will emulate. Seek out individuals who have a demonstrated commitment to diversity and inclusion.
Establish clear criteria and goals at the outset. What are the department's goals for this particular search? How will the ideal candidate be able to contribute to the department? What are the criteria by which candidates will be judged and how will we measure candidates using these criteria? These questions should be discussed before looking at candidate files so as to reduce the effect of bias, conscious or unconscious. If the department has a desire to hire an "underrepresented" person, clearly define what that means in the context of a specific subfield of the mathematical sciences, region of the United States, and current make-up of the institution and department.

Write a clear job ad that telegraphs the desires of your department and institution for greater diversity, whether it is to broaden participation in some way, promote social justice, or help create a more welcoming campus climate. Go beyond boilerplate equal employment opportunities statements required by your HR office. Make sure your criteria (see previous paragraph) are clearly conveyed. If any of your criteria have to do with candidates' ability to mentor underrepresented students or their interest in diversity and inclusion, consider asking candidates to make a statement about diversity or inclusion. Make it clear in the job ad whether that statement is encouraged or required, and where and how candidates are to include that in their application. Consider asking candidates to submit a separate diversity or inclusivity statement apart from the cover letter or teaching statement. As more and more institutions ask for these statements, whether you ask for them or not will send a signal to applicants about how much your institution cares about such things.

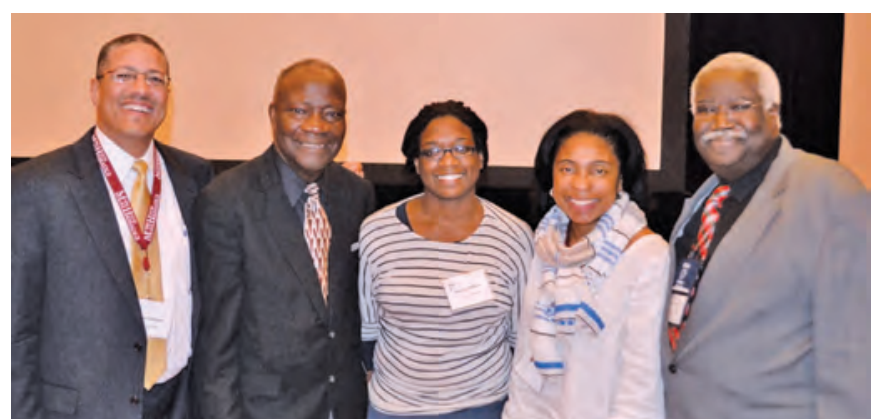

National Association of Mathematicians (NAM) Banquet at JMM 2014: Duane Cooper, Johnny Houston, Shelby Wilson, Ulrica Wilson, and Robert Bozeman ( $L$ to $R$ ).

Assemble the most diverse pool of candidates you can. Use personal networks to encourage people to apply. This is a crucial step if your department is seeking individuals who aren't already on the job market, and still helpful even if your position only targets beginning academics.

Advertise widely. While it may be true that most job seekers will find out about job opportunities in a few places, many women and people of color may also see your job ad in more targeted niches such as the Association for Women in Mathematics (AWM), SACNAS, National Association of Mathematicians (NAM), or the HBCU Career Center as a sign of your department's commitment to diversity. Attend AWM, SACNAS, and NAM events at the Joint 
Mathematics Meetings to make connections with people who might be able to help identify potential candidates. If you are hiring from the pool of recent PhDs, use the Annual Survey of the Mathematical Sciences published by the AMS to determine the demographic make-up of this pool. Periodically check your applicant pool to see how it compares to this overall make-up of all recent PhDs. If your department is conducting a nationwide search and has made no effort to diversify the applicant pool, the expected diversity of that pool should be similar to that of the national pool. Conversely, if your department has taken steps to assemble a diverse pool, this comparison can be one piece of evidence of the fruits of your department's efforts to administrators and other stakeholders. Strive to have an overrepresentation of women and people of color in your applicant pool.

Train committee members on equitable hiring practices. Seek out training for your search committee on how to minimize the impact of bias while reviewing files, what questions not to ask, how to create a welcoming and equitable on-campus interview protocol, and other related topics. In our experience, it is helpful to remind committee members about how cognitive shortcuts can lead to suboptimal decisions.

Eliminate bias as much as possible. All humans, even well intentioned ones, exhibit unconscious bias that can have significant consequences on searches. Therefore, search committees should take steps to eliminate bias, intentional and unintentional, as much as possible. One idea is to erase names from files. It's time-intensive and doesn't reduce bias completely but can reduce the effect of implicit associations that we might make based on names. Another idea is to make a round of decisions without looking at recommendation letters, as letters provide subjective information that is often difficult to interpret fairly and they privilege individuals who have access to influential people in their field. We strongly urge committees to randomize the order in which applicant files are read to avoid the unintentional bias that results when multiple committee members read files in the same order. When making a decision about a candidate, encourage committee members to articulate their reasons for arriving at that decision, preferably while making reference to the criteria for the search. When appropriate, codify those reasons into rules that can be applied fairly to other applicants.

Include candidates, rather than eliminate them, during the early stages of the search. Let's be honest: many of the candidates for a faculty position are not viable. Applicants might be in a field completely unrelated to the desired research area, or might have too little experience for a senior position, or might not be a permanent resident or US citizen for a government-funded position. To be efficient, searches are often structured so that the amount of time spent determining if an applicant is worthy of further consideration is less than the amount of time spent determining if an applicant is worthy to be invited for an interview. But, the rapidity of these decisions during the early stages of the search requires that committees exercise care to avoid inadvertently eliminating candidates from consideration, due to unintended bias or clerical error. We recommend that committees aim to have every applicant file read by more than one person, if possible, and to allow any committee member to include a candidate onto a list for further consideration, rather than allowing any individual to eliminate applicants from further consideration. Similarly, in later rounds of the search process, avoid allowing a single committee member to eliminate a candidate from further consideration without discussion.

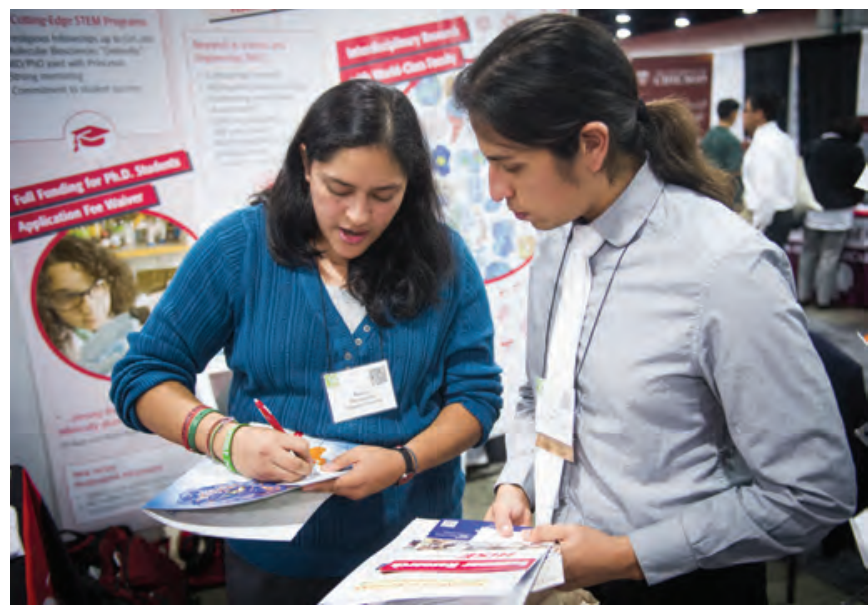

Students connecting with each other at the 2016 National Diversity in STEM Conference, Long Beach, CA, October 2016.

Gather data uniformly as it can be difficult to compare two candidates when there are different amounts or kinds of information about each person. If your committee will interview candidates using the phone or video conference, stick to one communication medium (phone or video) for everyone to avoid unintended bias [1, 2]. If your committee will interview candidates at the Joint Mathematics Meetings, try to have the same committee members present at all meetings. Agree on and use a common set of questions for all candidates.

Make the on-campus interview process welcoming and equitable. When finalists are brought to campus for interviews, the process should be just as much about the candidate finding out about the campus and department as the other way around. Ask finalists if there are specific groups of people that they would like to meet. Make sure all parties (especially students) involved in the search process know what kinds of things they aren't supposed to ask of finalists. However, if a finalist offers personal information (such as a desire for a certain kind of school for their children, or desire to participate in a particular faith community), make a note of that in case the candidate makes it to the offer stage. If candidates are expected to teach and/or give a research talk, provide uniform instructions and information to all finalists. Focus on what is clearly known about each finalist and avoid speculating about their reasons for applying, whether they would be willing to relocate, reasons for leaving their current job, etc. If a finalist is a woman or person of color, don't assume that they will be in such high demand that you won't be able to convince them to take the position. 
Be flexible and clear during the offer stage. Lobby your administration for any tactics that will increase the chances that your candidate will accept the offer. For example, flexibility on the start date, financial support for relocation, and spousal hires can help to sweeten the deal. Be responsive to any needs that the candidate might have expressed during the interview. Be clear about the parameters of the offer to avoid having to retract it.

Make a commitment to hire for success. Once a faculty member has been successfully hired, the department should commit itself to that person's success. Identify the right mentors and opportunities to allow that person to flourish. Make explicit and transparent the pathway to promotion and tenure.

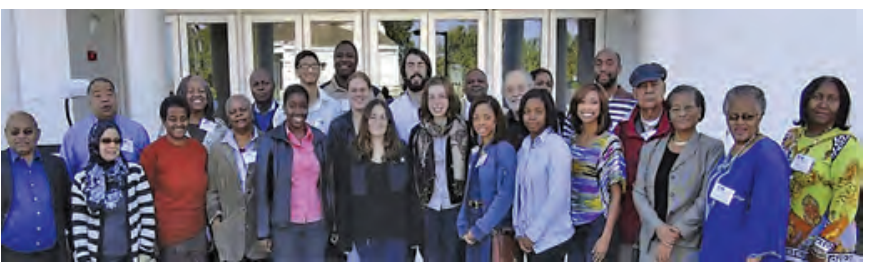

NAM Undergraduate MATHFest XXI at Dillard University in New Orleans in November 2011.

\section{Section 2. Best Practices for Faculty Who Are on Hiring Committees}

The previous section deals with practices that committees should consider to promote diversity and inclusion. The following section spells out some specific things that individuals serving on search committees can do.

Relationships matter. Since most of us academics will spend years at the same institution working with the same set of people, it is important to cultivate healthy relationships with our colleagues. Building and maintaining these relationships takes time and effort. Talking about race, bias, and privilege can be uncomfortable for many. It is important to keep in mind that each person is on their own journey with respect to diversity and inclusion. If you take the time to listen to your colleagues' life stories, they will be more likely to listen to yours. And, being able to truly listen to one another is the first step in being able to have conversations on difficult subjects.

To illustrate the importance of this idea, I (Darryl) will share an anecdote involving a colleague who is a diversity officer at a liberal arts college and who happens to be a woman of color. A senior faculty member, who happens to be a White male, made some inexplicably rude remarks to her one day. (The circumstances leading to these remarks aren't germane here.) She graciously reached out to the faculty member to get to know his life story and forged a connection with him. That faculty member, who had previously not been a champion for diversity, started attending all of the diversity-related events on campus. Coincidentally, that senior faculty member was also the chairperson for a faculty search committee. His leadership on the search committee and advocacy for diversity was a major reason that the department hired a woman of color for the position. This anecdote reminds me that the work of diversity is slow and laborious because it ultimately involves changing hearts and minds of individuals, who collectively enact an institution's policies and practices.

Calling in or out? If you encounter resistance or troublesome behavior from other committee members with regards to diversity and equity, think strategically about whether to "call in" or "call out." Calling out means bringing public attention to an individual's behavior that you find oppressive, racist, sexist, etc. Even when done so graciously, calling out often results in shaming and isolation of that individual. However, it can stop the behavior quickly and therefore can be effective in specific circumstances. Calling in involves a more personal approach, perhaps via a private conversation, to let the person know the impact of their words or actions and allow the person to take responsibility and seek restoration. The right approach to take will depend on the specific situation, but we generally counsel individuals to "call in" first. Calling someone out can lead to increased tension in committee meetings and in the department. Even if you eventually hire that amazing underrepresented candidate for your position, would you want to subject that person to a toxic work environment? Make healthy relationships a priority so that you can build a supportive culture in your department.

Practice self-care. Advocating for diversity is emotionally demanding work. To do this work over the long term, we need to develop self-awareness and mechanisms for taking care of ourselves. Surround yourself with trusted colleagues and allies. Recognize that there will be setbacks, so celebrate your successes, no matter how small!

\section{Section 3. Helpful resources}

Here are some of our favorite resources for search committees:

- Searching for Excellence and Diversity: A Guide for Search Committees by Eve Fine and Jo Handelsman (published by WISELI: Women in Science and Engineering Leadership Institute, available online or can be ordered in print at wiseli.engr.wisc.edu/ searchguidebooks.php)

- Two resources created by the University of Washington:

- Handbook of Best Practices for Faculty Searches www.washington.edu/diversity/faculty-advancement/handbook/.

- An online video and facilitation guide entitled "Interrupting Bias in the Faculty Search Process" www. engr.washington . edu/lead/biasfi 1m//. - A great primer on implicit bias by Kimberly Pappilon: https://equaljusticesociety.org/law/ implicitbias/primer/.

We believe that training on reducing implicit bias is one of the most fruitful things that search committees can do. Most people don't want to be racist or sexist and want to do the right thing. However, it is important to realize that our human brains are wired to draw conclusions based on data and that those conclusions sometimes lead to sub-optimal decisions. For example, because most US physicists are White and male, most Americans are more likely to associate being a physicist with being White and 
male. This association doesn't imply that we're racist and sexist, but we should recognize that our implicit biases often have the same effects as overt racism and sexism.

The best way to mitigate our implicit biases is to slow down and be more deliberate in our decisions. Search committee members often have the unpleasant task of reading lots of applicant files in a short amount of time. The faster we force our brains to make these decisions, the more likely our implicit biases will lead to suboptimal decisions. Other ways to mitigate implicit biases include (1) accepting that you are susceptible to the influence of implicit bias and associations, (2) intentionally cultivating relationships with individuals who are counter-examples to your implicit associations, (3) minimizing distractions when reviewing applications, (4) stopping periodically to check whether you are applying your criteria equitably, (5) looking holistically at a candidate using all available information instead of focusing on specific pieces of information.

Because mathematicians tend to like rigorous research studies and data, it might be helpful to present search committees with some research studies that validate the existence of implicit bias. Harvard's Project Implicit $^{3}$ is a great way to learn about implicit associations and to determine the strength of one's own implicit associations. Daniel Kahneman's book Thinking, Fast and Slow summarizes a lot of the research on how humans arrive at irrational conclusions, especially when forced to think quickly.

We have also found Wikipedia's list of cognitive biases helpful because it enables committee members to name the specific mechanisms behind our implicit biases ${ }^{4}$ and associations. For example, anchoring is a cognitive bias in which we tend to give disproportionately more weight to the first piece of information that we receive when making a decision. In the context of reading applicants' files, anchoring happens when we scan files quickly and latch on to the applicant's name (and our perceptions of their gender and ethnicity) or graduate school. Another relevant cognitive shortcut is confirmation bias, which is the tendency to interpret and recall information that aligns with one's preexisting beliefs about an applicant.

Mitigating implicit bias is a necessary, but not sufficient step, to create a more equitable and welcoming academy. We still need to deal with overt forms of discrimination and bias, institutional racism and sexism and other -isms, and underrepresentation in the mathematical sciences. We advocate for implicit bias training because it helps put people at ease, is effective, and is a good entry point to deeper discussions about equity and inclusion.

Broader participation in the mathematical sciences won't happen naturally-our hiring practices must be intentionally designed to promote diversity and inclusion if we want to make real progress toward that goal [6]. Not all of these strategies in this article may be appropriate for your particular situation. It is our hope that these

$3 \longdiv { \text { implicit.harvard.edu } }$
4 https://en.wikipedia.org/wiki/List_of_cognitive_bi-
ases
5 @dyongand@aSumunLPendakur

WISELI: Women in Science and Engineering Leadership Institute ${ }^{\circledR}$

\section{Top Ten Tips for Searching for Excellence and Diversity as detailed in WISELI's guidebook, Searching for Excellence and Diversity 6}

1. Build a diverse committee and ensure that all members understand the committee's role in the search process.

2. Build rapport among committee members by creating an environment of collegiality, respect, dedication, and open-mindedness. Ensure that all members play meaningful roles in the process.

3. Establish expectations and ground rules for such items as attendance, active involvement, decision-making, confidentiality, treatment of candidates, and more.

4. Air views about diversity, discuss ideas about excellence, and develop a shared understanding of what diversity and excellence mean for a particular search.

5. Recruit a diverse applicant pool by searching broadly and inclusively. Save sifting and winnowing for later.

6. Recruit aggressively by making personal contact with potential applicants, advertising in publications targeted to underrepresented groups, and communicating with organizations and people who can refer you to potential applicants.

7. Learn about research on unconscious or implicit biases and assumptions and their influence on your evaluation of applicants.

8. Question the objectivity of your own judgments and learn about other ways to mitigate bias. Implement policies and practices that can reduce the influence of unconscious or implicit bias.

9. Ensure that every candidate interviewed-whether hired or not-is respected treated well before, during, and after interviews and visits.

10. Maintain communication with your final candidates until an offer is accepted.

${ }^{6}$ Eve Fine and Jo Handelsman. Searching for Excellence \& Diversity, ${ }^{\circledR}$ National Edition. Copyright (C) 2012 by WISELI and the Board of Regents of the University of Wisconsin System. Reproduced with permission.

ideas will spur conversations at your institution and that this article will empower you and your colleagues toward greater inclusion, equity, and ultimately, societal change for the better.

If you have positive experiences using other pro-diversity faculty search practices, please share them with us on Twitter. ${ }^{5}$ 
We'd like to thank Ron Buckmire, Dagan Karp, Arnold Kim, David Kung, Rachel Levy, and anonymous referees for their helpful comments.

\section{References}

[1] BeHREND, TARA, STEVEN TOADDY, LORI FOSTER THOMPSON, and DAVID J. SHAREK. The effects of avatar appearance on interviewer ratings in virtual employment interviews. Computers in Human Behavior 28, no. 6 (2012): 2128-33.

[2] BlACKSMITH, NiKKI, JON C. WillFORD, and Tara S. Behrend. Technology in the Employment Interview: A Meta-Analysis and Future Research Agenda. Personnel Assessment and Decisions 2.1 (2016): 12-20.

[3] GRIFFIN, KIMBERLY A. Black Professors Managing Mentorship: Implications of Applying Social Exchange Frameworks to Analyses of Student Interactions and Their Influence on Scholarly Productivity. Teachers College Record 114.5 (2012): n5.

[4] LANDIVAR, LIANA CHRISTIN. Disparities in STEM employment by sex, race, and Hispanic origin. Education Review 29.6 (2013): 911-22.

[5] SMITH, DARYL. Diversity's Promise for Higher Education: Making it Work. John Hopkins University Press, 2009.

[6] Smith, Daryl, Caroline S. Turner, NANA Osei-Kofi, and SANDRA RICHARDS. Interrupting the usual: Successful strategies for hiring diverse faculty. The Journal of Higher Education 75, no. 2 (2004): 133-60.

[7] TAYlor, Teresa E., JefFrey F. Milem, and Arthur F. COleMAN (2016). Bridging the research to practice gap: Achieving mission-driven diversity and inclusion goals. New York, NY: College Board. Available at https://www.aacu.org/ node/16225.

[8] TURNER, CAROLINE SOTELLO VIERNES, JUAN CARLOS GONZÁLEZ, and J. LUKE WOOD. Faculty of Color in Academe: What 20 Years of Literature Tells Us. Journal of Diversity in Higher Education 1.3 (2008): 139.

\section{Photo Credits}

Photo of AWM Panelists at JMM courtesy of AWM.

Photo of Exhibit Hall connections courtesy of SACNAS.

Photo of NAM participants courtesy of NAM.

Photo of MathFest courtesy of NAM.

Photos of Darryl Yong and Sumun Pendakur courtesy of Harvey Mudd College.

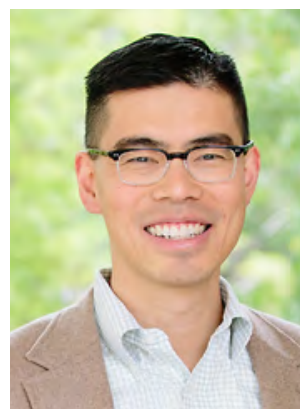

Darryl Yong

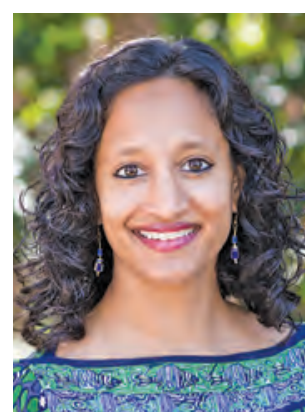

Sumun Pendakur

\section{ABOUT THE AUTHORS}

Darryl Yong's scholarly activities focus on the retention and professional development of secondary school mathematics teachers and improving undergraduate mathematics education. Darryl serves on the Steering Committee for Math for America Los Angeles and helps to design professional development for its Fellows.

\section{Sumun Pendakur serves as} a consultant, speaker, and facilitator regionally and nationally, helping campuses, nonprofits, and other organizations build capacity for cultural competence, social justice, and equitable practices. She is a scholar-practitioner whose research interests include critical race theory, Asian American and Pacific Islander students, and institutional transformation. 